\title{
MEDIA MOTION-BASED RESOURCE DISTRIBUTION FOR MOBILE VIDEO NETWORKING
}

\author{
U. S. Ukommi* \\ Department of Electrical and Electronic Engineering, AKWa Ibom State University, IKot AkPaden, \\ MKPAT ENIN, AKWA IBOM STATE, NIGERIA. \\ Email address: uukommi@yahoo.com
}

\begin{abstract}
Wireless video communication is challenging due to vulnerability of media bitstreams to channel distortions. Investigation has been carried out on wireless video channel under tight networking resource budget. One of the challenges is the impact of channel errors on the quality of media streams with high motion activity. Motion activity in this context defines the magnitude of activity displacement in video sequence. Based on the analysis, Media Motion-based Resource Distribution (MRD) is proposed to maximize the average received video quality over wireless system, by regulating the resource distribution of the media streams based on their motion activity characteristics. Experimental results demonstrate that the proposed scheme can improve the average received video quality performance under tight resource constraints budget.
\end{abstract}

Keywords: Wireless video communication, resource constraints, received video performance, media motion.

\section{INTRODUCTION}

Wireless networks provide platform for immersive video distribution. Wireless video communication is more prone to channel errors compared to that of a wired channel due to varying channel conditions and resource constraints. A typical video communication system consists of the video source encoder, channel encoder and decoder blocks. The video encoder such as H.264/AVC and H.265 HEVC perform video compression and support error resilience features. In wireless video communication, resource constraints pose major challenges for effective video communication with improved received video quality. Wireless technology such as Mobile WiMAX, $4 \mathrm{G}$ and $5 \mathrm{G}$ network $[1,2]$ provide medium for video communication services and applications at anytime, anywhere to the target regions and clients. Mobile WiMAX, which uses Orthogonal Frequency Division Multiplexing Access (OFDMA) technology, plays a significant role in the fourth generation and fifth generation wireless access systems due its flexible resource allocation and inherent tolerance against multipath fading experienced by mobile users. The platform supports centrally-controlled resource distribution scheme for efficient video services delivery. OFDMA divides the available resources into a number of subchannels, and supports Convolutional Turbo Coding (CTC) channel coding for improved quality of video services. Modulation schemes such as Quadrature Phase Shift Keying (QPSK) and Quadrature Amplitude Modulation (QAM) are also supported in OFDMA with variable channel coding rates which offer channel protection and throughput for video services. The performance of a wireless transmission is determined by the Signal-to-Noise Ratio (SNR). SNR takes the relative factors such as path loss, cable loss into account. The relationship between SNR and BER is approximated by:

$$
\mathrm{BER} \leq C_{1} \mathrm{e}^{-\left(\frac{\mathrm{C}_{2} \mathrm{SNR}}{\mathrm{M}-1}\right)}
$$

where the constants $C_{1} \approx 0.2$ and $C_{2} \approx 1.5$ [3]. $M$ represents constellation state of the modulation scheme. Wireless video communication applications and services are becoming indispensable means of communication in the society due to the mobility and capability of satisfying client demand at "anywhere"

* Author, tel: +234- $703-246-5163$ 
and "anytime", and present a lucrative source of revenue generation for mobile video service providers. However, there are many challenges in wireless communication channels such as power and bandwidth constraints. These factors influence the quality of wireless video services. Thus, this paper focuses on developing an efficient resource distribution strategy to maximize the overall received video quality for a set of media streams under tight constrained resource budget. This research work harness the video characteristic in the resource distribution strategy. The rest of the paper is organized as follows. Section 2 presents related work. Section 3 models media motion characteristic which is harnessed in the resource distribution. Section 4 describes the proposed system. Section 5 explains the simulation methodology. Section 6 presents the results findings and performance comparison of the proposed scheme and conventional technique. Finally, the paper is concluded in Section 7.

\section{RELATED WORK}

Different resource distribution schemes have been discussed in literature such as sub-optimal joint subcarrier and power allocation algorithm for multiuser OFDM, which adopt water-filling mechanism [4], Optimal Power Allocation in Multiuser OFDM systems [5] and Optimal Power Allocation for Multirate Systems with interference cancellation [6]. Unequal Power Allocation for Scalable Video Transmission, where base layers of scalable video are allocated more transmit power compared with the enhancement layer packets, is proposed in [7]. Adaptive Power Allocation and Call Admission Control in Multirate Service WiMAX Access Networks, which allocates more power resources to the users with higher subscription tariff has been discussed in [8]. An Unequal Error Protection Scheme for Object-based Video Communications, where different quantization parameters are allocated for coding of each object is discussed in [9]. However, the discussed schemes in the literature do not consider media motion characteristic in video networking process. Thus, the objective of the research work, which envisages mechanism of harnessing media motion characteristic in video networking to improve received video quality under a limited network resource budget. The significant optimization strategy presented in this paper is the systematic exploitation of the media motion characteristics of the media streams in the video quality performance enhancement. One of the significant advantages of OFDMA system is the orthogonality characteristic among the subcarriers, which mitigates intra-cell interference. Another key benefit of OFDMA air interface is the frequency reuse capability, which mitigates inter-cell interference. Several advanced interference management schemes for fourth generation cellular standards are discussed in literature. Such interference management schemes include sectorisation, power control and regulated maximum transmit power level that can be applied to media streams. The details on these interference management techniques are discussed in $[10,11]$. Assuming, a downlink transmission scenario where the effect of interference is negligible, the maximum base station transmit power becomes a significant parameter in determining the overall system performance. To reduce complexity, the proposed system considers a downlink scenario and assumes negligible interference. Figure 1 , presents typical wireless video networking system architecture.

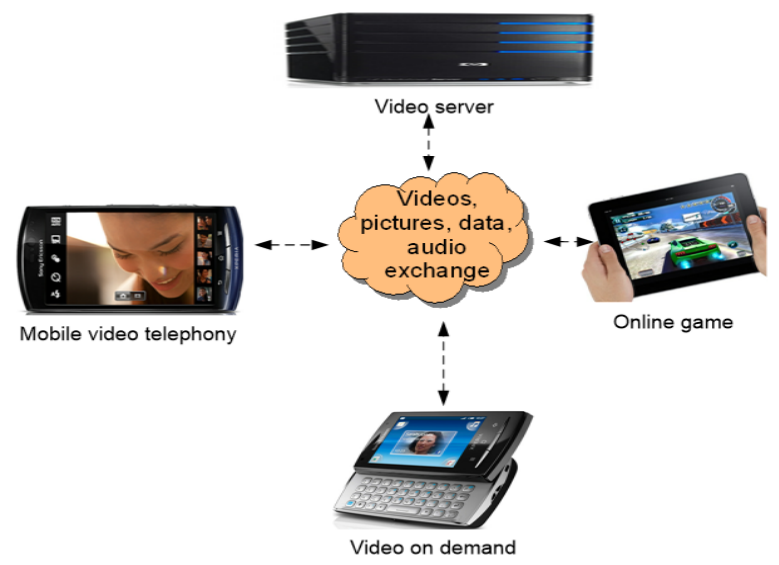

Figure 1: Typical wireless video networking system architecture.

\section{MODELING OF MEDIA MOTION}

Quantitatively, the media motion of media streams in this research is based on feature points of the objects in the video scene. The media motion is analysed using the optical flow algorithm of Lucas and Kanade [12]. The measurement of the media motion is characterized by identification of significant feature points. Feature point in this concept is defined as an interesting point of the object in video scene that can be easily detected and tracked from frame to frame. Types of image feature points include edges, blobs, ridges and corners. Corners can be defined as interest points in a video scene 
that can be efficiently detected and a point where the edge direction changes rapidly [13]. In the measurement of the media motion characteristic, the object is first detected and analyzed to determine the changes in motion. Feature point (p) relative difference in terms of pixel displacement between successive video frames quantifies Motion Vector (MV) of the feature point. MV is represented by vertical and horizontal components $M V(X i, Y j)$. The MV of a feature point $(p)$ in a video frame is given by:

$$
M V_{\mathrm{p}}=\sqrt{X_{P}{ }^{2}(\mathrm{i}, \mathrm{j})+Y_{P}{ }^{2}(\mathrm{i}, \mathrm{j})}
$$

The media motion $\left(\Delta_{\mathrm{L}}\right)$ of a frame is calculated as the sum of all MVs in the video frame:

$$
\Delta_{\mathrm{L}}=\sum_{i=0}^{P-1}\left|M V_{p}(X i, Y j)\right|
$$

where $M V_{p}(X i, Y j)$ represents the motion vector of the frame at feature point ( $p)$. A typical video scene consists of objects characterized by spatial characteristics (number and shape of objects) and temporal characteristics. The lower the temporal resolution of the video sequence the higher the motion intensity because of the large difference in motion between successive video frames compared to higher temporal resolution, where the difference in motion between successive video frames is relatively lower [14]. Media motion of a video frame is proportional to spatial resolution $(R)$ of the video frame and inversely proportional to the temporal $(\mathrm{T})$ resolution of the video sequence [15]. Thus, the total average media motion characteristic over a given video sequence is normalized by the spatial and temporal resolutions to maintain consistency across different video sequences:

$$
\Delta_{\mathrm{S}}=\sum_{i=0}^{L-1} \Delta_{\mathrm{L}} \times \frac{R}{T}
$$

where $\Delta_{\mathrm{s}}$ is the media motion characteristic of the video sequence over a given number of video frames $L$. The frame rate and spatial resolution of the video sequence are represented by $T$ and $R$, respectively. Figure 2 shows media motion characteristic of Akiyo, Foreman, and Soccer test video sequences with media motion characteristic across test video frames. The graph depicts that each test video sequence has unique media motion characteristic, which is harnessed in the proposed system for effective mobile video networking.

\section{PROPOSED SCHEME}

In many wireless video communication scenarios, the primary constraint for reliable communication with good video quality performance arises from transmit resource constraints [16]. Video applications and services require high bitrates to attain good quality. However, at higher data rate requirements, limited bandwidth and tight transmit power constraints, it becomes challenging with the limited wireless resources to support improved received video quality [17]. Media motion-based resource distribution proposes an efficient transmit resource distribution strategy for improved video services based on the media motion characteristics of media streams.

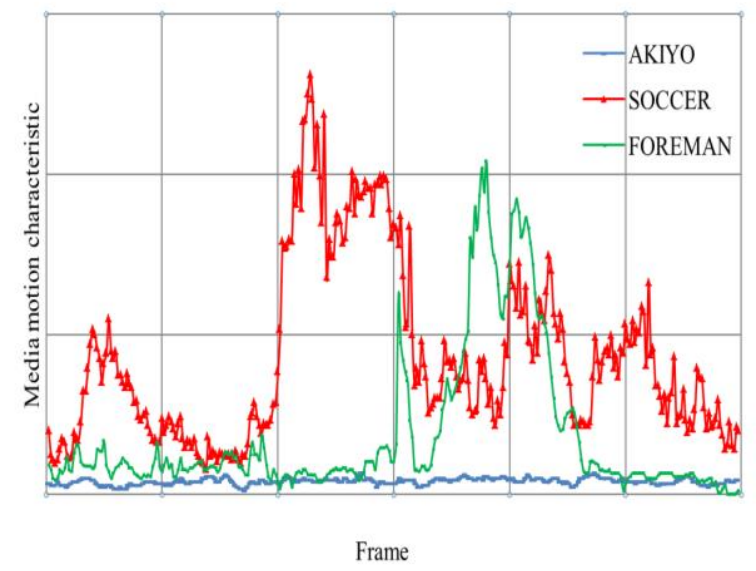

Figure 2: Media motion characteristic of Akiyo, Soccer and Foreman test video sequences.

The objective of the proposed scheme is to improve the average received video quality performance for a set of media streams under constrained wireless network resources. The total video distortions consist of source distortion [18] and channel distortion [19]. It becomes difficult under fixed constrained network budget and low bandwidth availability to support error protection scheme for improved received video quality. Thus, a need to research and design a system that maximizes the usage of the limited wireless resources to improve the average received quality of wireless video services. However, in order to devise an efficient model, media motion-based resource distribution framework jointly investigates the impact of transmission resource constraints and media motion characteristics of media streams on the received video quality. The framework of the proposed scheme consists of media server which stores the media contents. The media encoder performs 
compression and source error functionalities [20]. The analyser evaluates motion levels of the media streams. The motion-based module distributes transmit resources to media streams based on the motion information of the media stream, derived from the analyzer. The proposed scheme aims at maximizing the average received video quality of the media streams by adapting network resources distribution based on the media motion characteristic of the media content, distortion level of the media stream and the prevailing channel conditions. To demonstrate efficacy of the proposed scheme, a simple approach of Equal Resource Distribution (ERD) technique, where all media streams are treated fairly with equal network resource allocation are compared with the proposed system where media streams network resource allocation are carried out based on media motion characteristics.

\section{SIMULATION}

Figure 3, shows the simulation set up. The media server stores the test media contents. The media motion characteristic is analysed using motion analyser and the H.264/AVC encoder codes the media contents.

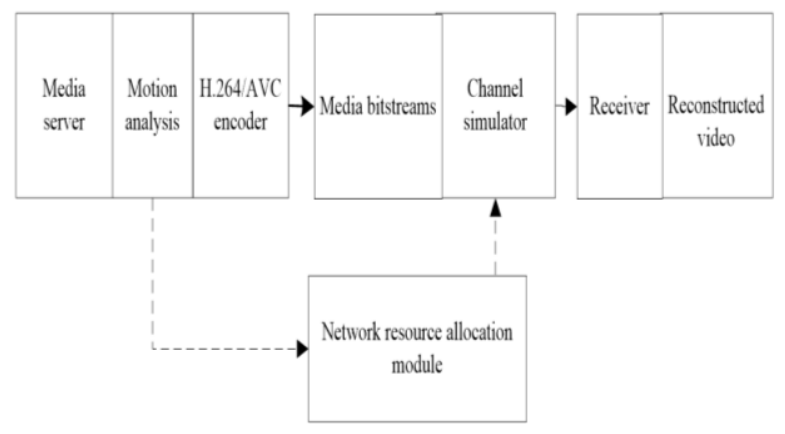

Figure 3: Simulation Set up

The test media samples are classified into classes; high motion characteristic and low motion characteristic based on the motion characteristic of the test media samples. It clustered test media samples that have similar motion characteristics into group. For consistency and accurate assessment of the impact of media motion on the received video quality, all test video sequences used in the simulation process are in Common Intermediate Format (CIF) of $352 \times 288$ pixels for mobile video services and applications. The source coding of the test media sequences is performed using the H.264/AVC reference software, version 15.1 (JM
15.1) [21]. Foreman, Akiyo and Soccer standard test sequences are used in the research. In order to enhance accurate assessment of the effect of the activity and power constraint, the source rate for the test video sequences is maintained at $0.768 \mathrm{Mbps}, 30$ frames per second, group of picture size of 8 for all the test video sequence. Each video sequence has a total number of 150 frames processed in IPPP... format (first frame of each sequence is intra-coded, followed by P-frames), with Content Adaptive Binary Arithmetic Coding (CABAC) technique. The slice is encapsulated in RTP/UDP/IP [22] for robust transmission through the protocol stack of WiMAX wireless system. At the Network Abstraction Layer (NAL), slice output of the VCL is placed in NALU prior to transmission. For optimization of the payload header [23] and reduction of high loss probability the NALU length is fixed at 512 bytes for all test video sequences. RTP transport is augmented by a control protocol (RTCP) [24] for monitoring of the data delivery and provision of feedback on the reception quality. The same parameter settings are maintained for all of the test video sequences in order to enhance accurate analysis of the impacts of motion intensity level and power constraints on received video quality.

The channel simulator performs channel coding using simulated mobile channel conditions for 16QAM, $1 / 2$ modulation scheme with a range of SNR levels. The properties of the error traces include downlink subframe of 30 subchannels $\times 13$ time symbols, giving a total of 390 slots. The error patterns are obtained by comparing all the data bits within the original data slot to the transmitted data slot. If there is any bit error within the data slot, it is then declared as an error. The simulator models a time varying channel including the effects of multipath for the ITU Vehicular A scenario. The channel coding and decoding have also been taken into account in the simulator. The error traces with the similar SNR are used to corrupt the media streams transmitted through the simulator. The system level simulations are performed to investigate the impacts of activity and resource constraints on the received video quality using lookup tables. The look-up tables translate the bit error rate information into distortion levels, which are used to distribute the power among the competing media streams. The network resource distribution is carried out by initially allocating equal minimum transmit power level for all media streams, then incremented 
based on the activity characteristic and the distortion levels of the media streams. Table 1 , shows the system parameter configuration for the simulation. The experimental results were averaged over repeated twenty simulations in order to obtain more stable results and evaluate accurately the performance of the proposed scheme. At the receiving end, the corrupted media streams are demodulated and decoded. H.264/AVC decoder reference software version 15.1 (JM 15.1) is used for source decoding. The error concealment with frame copy mode is employed for concealment of corrupted video packets.

Table 1: System Parameter Configuration

\begin{tabular}{ll}
\hline System & Parameters \\
\hline Encoder & H.264/AVC encoder \\
Target bitrates & 768Kbps \\
Video format & $4: 2: 0$, YUV \\
GOP & 8.0 \\
Frame & 300 \\
Frame rate & $30 \mathrm{fps}$ \\
Packet seize & 512 bytes \\
Channel type & Time varying channel \\
Activity level & Compute activity level of \\
analyser & video contents \\
Modulation \& FEC & $16 Q \mathrm{AM}, 1 / 2$ \\
Channel coding & Convolutional Turbo Coding \\
Bandwidth & (CTC) \\
Carrier frequency & 2.5 GHz \\
& PUSC \\
Duplexing scheme & TDD \\
FFT size & 1024 \\
Channel model & ITU Vehicular A \\
Path loss model at & ITU-R \\
distance R(km) & H.264/AVC decoder with \\
& error concealment \\
Decoder & algorithm \\
\hline
\end{tabular}

When a packet is lost, the RTP sequence number enables the decoder to identify the lost packet such that the location of the corrupted packet in a frame is identified and concealed. The same decoder parameter setting is maintained for all the test video sequences. Peak Signal-to-Noise Ratio (PSNR) is used to measure the level of distortion on the received video quality.

As a measure of the objective function which can be defined as maximizing the average received video quality among the media streams within the tight power constraints, PSNR [25] is employed to measure the efficiency of the proposed system in terms of the average received video quality performance of the media streams at the receiver. PSNR is employed in measuring the received video quality performance because it is widely employed in the field of video quality performance measurement. It is easy and fast in terms of reduced computational complexity and saves time. The lookup table generated experimentally with 16QAM modulation and $1 / 2$ FEC scheme is presented in Table 2, for simulation purpose. It consists of a range of network resource levels in terms of SNR and the corresponding distortion levels for the test media sequences. In the simulation, the system performance is tested with Soccer, Foreman and Akiyo test sequences at different Signal to Noise Ratio (SNR) levels as represented in Table 2.

\section{RESULT AND DISSCUSSION}

Experiments are carried out to evaluate the efficiency of the proposed technique in terms of received video quality performance. The effectiveness of the system is measured in PSNR values from the reconstructed media frames with the application of error concealment algorithm, which replaces corrupted frames with those from the previously correctly decoded frames. Table 3 , shows the overall comparative performance of the proposed media motion-based Resource Distribution scheme and the conventional scheme, which allocates equal resources to media streams without consideration of the impact of motion characterization.

Table 2: Lookup table

\begin{tabular}{lcrrr}
\hline SNR $(\mathrm{dB})$ & BER & \multicolumn{1}{c}{$\begin{array}{l}\text { Soccer } \\
(\mathrm{dB})\end{array}$} & $\begin{array}{c}\text { Foreman } \\
(\mathrm{dB})\end{array}$ & \multicolumn{1}{l}{$\begin{array}{l}\text { Akiyo } \\
(\mathrm{dB})\end{array}$} \\
\hline 9.95 & $7.74 \times 10^{-3}$ & 25.42 & 26.49 & 38.70 \\
10.50 & $3.47 \times 10^{-3}$ & 29.65 & 23.04 & 41.76 \\
11.05 & $1.40 \times 10^{-3}$ & 32.67 & 30.66 & 44.66 \\
11.60 & $5.20 \times 10^{-4}$ & 33.95 & 35.47 & 50.16 \\
12.15 & $1.74 \times 10^{-4}$ & 36.14 & 36.05 & 50.47 \\
12.70 & $6.15 \times 10^{-4}$ & 36.37 & 37.39 & 50.48 \\
13.25 & $2.43 \times 10^{-4}$ & 36.78 & 38.32 & 51.08 \\
\hline
\end{tabular}

Table 3: Quality performance comparison of ERD and the proposed system MRD schemes

ERD Scheme MRD Scheme

The overall quality performance of the proposed MRD scheme and ERD scheme are compared in terms of the average reconstructed media quality (PSNR) performance under tight network resource constraints. From Table 3 the average received media quality performance of the media streams 
under MRD scheme outperforms the conventional scheme. The enhancement is more conspicuous with the foreman test video sequence which recorded 29.66dB with ERD approach and 35.47dB with the proposed MRD scheme as shown in Table 3. The quality performance of Soccer test media sequence quality performance also improves from $28.90 \mathrm{~dB}$ under ERD to $31.99 \mathrm{~dB}$ while using the proposed scheme. The reason for the improved performance is due to the fact that the proposed MRD system enhances the protection level of the sensitive media streams more efficiently and mitigates the impact of channel errors on the reconstructed video quality. It has also been observed that it is difficult to reconstruct accurately the lost media data in the media stream with relative high media motion characteristic which results in poorly reconstructed media quality performance. The visual and quantitative quality performance comparison of the ERD and MRD are presented in Table 3.

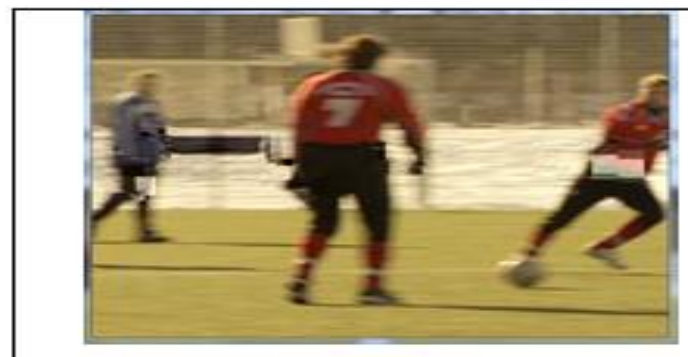

$28.90 \mathrm{~dB}$ (Soccer test sample)

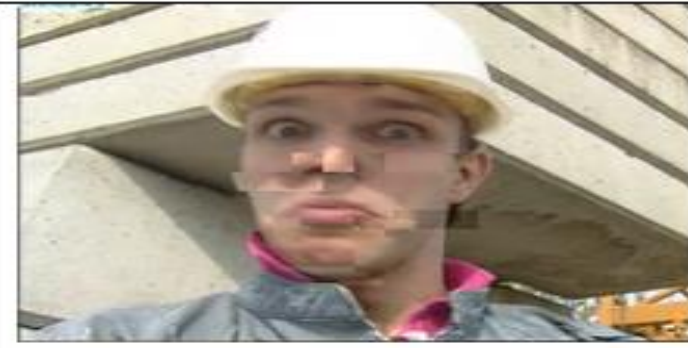

29.66dB (Foreman test sample)

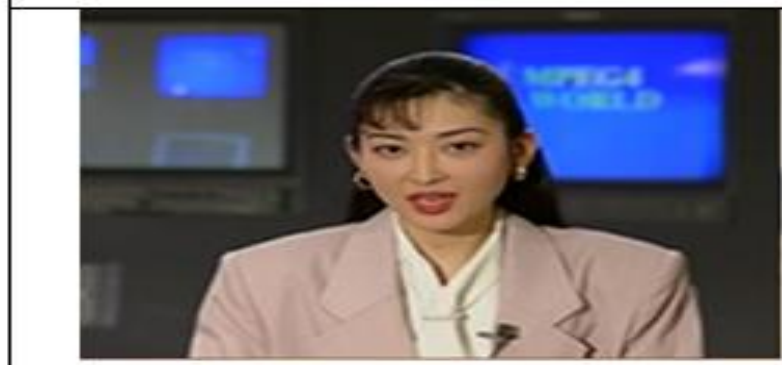

44.65dB (Akiyo test sample)

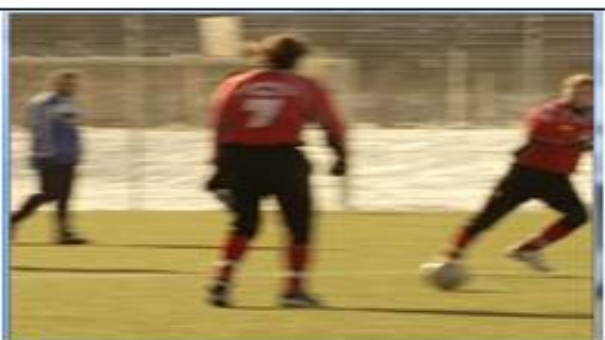

31.99 dB (Soccer test sample)

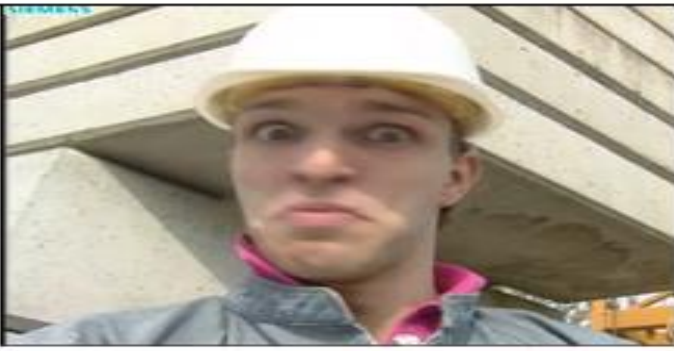

$35.57 \mathrm{~dB}$ (Foreman test sample)

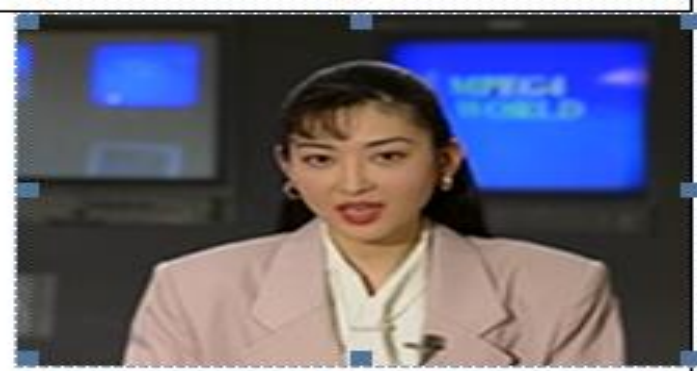

49.47dB (Akiyo test sample)

\section{CONCLUSION}

The impact of media motion characteristic on received media quality performance has been investigated. A novel solution, Media Motion-based Resource Distribution (MRD) scheme for wireless mobile video networking, which improves the average received video quality performance constrained network resources, is proposed. The simulation results show that the proposed system performance is capable of improving the average received media quality. To further improve the system performance, future work jointly investigates more advanced parameters and applications scenarios in developing more efficient scheme for wireless video networking applications. 


\section{REFERENCES}

[1] 5G World Forum, "IEEE Future Networks", 2019. https://ieee-wf-5g.org/

[2] S. Singh and P. Singh, "Key Concepts and Network Architecture for 5G Mobile Technologies, International journal of Sceintific research Engineering and Technologt, 2012.

[3] R. Jain, S.-I. Chakchai, and A. k. Al Tamimi, "System-level modeling of IEEE 802.16E mobile wimax networks: Key issues," Wireless Communications, IEEE, vol. 15, pp. 73-79, 2008.

[4] C. Mohanram and S. Bhashyam, "A sub-optimal joint subcarrier and power allocation algorithm for multiuser OFDM," Communications Letters, IEEE, vol. 9, pp. 685-687, 2005.

[5] S. Zukang, J. G. Andrews, and B. L. Evans, "Optimal power allocation in multiuser OFDM systems," in Global Telecommunications Conference, 2003. GLOBECOM '03. IEEE, 2003, pp. 337-341 Vol.1.

[6] G. Zihua and K. B. Letaief, "Optimal power allocation for multirate CDMA systems with interference cancellation," in Communications, 2001. ICC 2001. IEEE International Conference on, 2001, pp. 1885-1889 vol.6.

[7] Z. Ahmad, S. Worrall, and A. Kondoz, "Unequal power allocation for scalable video transmission over WiMAX," in Multimedia and Expo, 2008 IEEE International Conference on, 2008, pp. 517-520.

[8] R. Bo, Q. Yi, and C. Hsiao-Hwa, "Adaptive power allocation and call admission control in multiservice WiMAX access networks [Radio Resource Management and Protocol Engineering for IEEE 802.16]," Wireless Communications, IEEE, vol. 14, pp. 14-19, 2007.

[9] S. Nasir, S. Worrall, M. Mrak, and A. M. Kondoz, "An unequal error protection scheme for object based video communications," in Consumer Electronics, 2008. ISCE 2008. IEEE International Symposium on, 2008, pp. 1-4.

[10] N. Himayat, S. Talwar, A. Rao, and R. Soni, "Interference management for $4 \mathrm{G}$ cellular standards [WIMAX/LTE UPDATE]," Communications Magazine, IEEE, vol. 48, pp. 8692, 2010.

[11] L. Taeyoung, Y. Jisun, L. Sangtae, and S. Jitae, "Interference management in OFDMA Femtocell systems using Fractional Frequency Reuse," in Communications, Circuits and Systems (ICCCAS), 2010 International Conference on, 2010, pp. 176180.

[12] D. Fleet and Y. Wiess, "Optical Flow Estimation in Paragios. ," Handbook of Mathematical Models in Computer Vision, Springer, 2006.
[13] Jianbo and et al, "Good features to track " IEEE Computer Society Conference on Computer Vision and Pattern Recognition, 1994.

[14] I. E. G. Richardson, "H.264 and MPEG-4 Video Compression," John Wiley and Sons Limited. West Sussex, England, 20032003.

[15] G. Nur, H. K. Arachchi, S. Dogan, and A. M. Kondoz, "Advanced Adaptation Techniques for Improved Video Perception," Circuits and Systems for Video Technology, IEEE Transactions on, vol. 22, pp. 225-240, 2012.

[16] R. G. Gallager, "Energy Limited Channels: Coding, Multiacces and Spread Spectrum," Tech. REP., MIT. LIDS-P-1714, November 1987.

[17] O. Oyman, J. Foerster, T. Yong-joo, and L. SeongChoon, "Toward enhanced mobile video services over WiMAX and LTE [WiMAX/LTE Update]," Communications Magazine, IEEE, vol. 48, pp. 6876, 2010.

[18] L. Ke-Ying, Y. Jar-Ferr, and S. Ming-Ting, "RateDistortion Cost Estimation for H.264/AVC," Circuits and Systems for Video Technology, IEEE Transactions on, vol. 20, pp. 38-49, 2010.

[19] W. Yao, W. Zhenyu, and J. M. Boyce, "Modeling of transmission-loss-induced distortion in decoded video," Circuits and Systems for Video Technology, IEEE Transactions on, vol. 16, pp. 716-732, 2006.

[20] T. Wiegand, G. J. Sullivan, G. Bjontegaard, and A. Luthra, "Overview of the H.264/AVC video coding standard," Circuits and Systems for Video Technology, IEEE Transactions on, vol. 13, pp. 560-576, 2003.

[21] A. M. Tourapis, "Joint Video Team (JVT) of ISO/IEC MPEG \& ITU-T VCEG (ISO/IEC JTC1/SC29/WG11 and ITU-T SG16 Q.6)," Geneva, http://iphome.hhi.de/suehring/tml/, February 2009.

[22] S. Wenger, M. Hannuksela, T. Stockhammer, M. Westerland, and D. Singer, "RTP Payload Format for H.264 Video," IETF, RFC 3984, February 2005.

[23] S. Jung, "Effect of Robust Header Compression (ROHC) and Packet Aggregation on Multi-hop Wireless Mesh Networks," IEEE 6th International Conference on computer and Information Technology (CIT'06), 2006.

[24] S. C. H. Schulzrinne, R. Frederick, V. Jacobson, "RTP: A Transport Protocol for Real-Time Applications, RFC 3550 Internet-Draft," IETF Network Working Group, July 2003.

[25] M. Vranjes, S. Rimac-Drlje, and K. Grgic, "Locally averaged PSNR as a simple objective Video Quality Metric," in ELMAR, 2008. 50th International Symposium, 2008, pp. 17-20. 